THE USE OF REDUNDANCY IN INTERFEROMETRY A COMPARISON OF REDUNDANCY AND SELFCAL

\author{
MARK WIERINGA \\ Leiden Observatory, P.O. Box 9513, 2300 RA Leiden, The Netherlands
}

\begin{abstract}
Many synthesis arrays currently in use have at least a few redundant baselines which can be used to derive model-independent telescope corrections. We discuss the relative merits of redundancy-based versus standard selfcalibration methods with respect to sensitivity to noise and interference. We then derive an approach to optimally combine the two methods for partially redundant arrays
\end{abstract}

\title{
USING REDUNDANCY
}

Redundant baseline calibration uses the fact that for some types of synthesis arrays, especially one-dimensional arrays with many equally spaced telescopes (like e.g., the Cambridge, Penticton and Westerbork arrays), the same baseline vector occurs more than once. Because the true visibilities on equal baselines are equal the measured visibilities contain information on the complex telescope gains (instrumental and atmospheric effects). For each redundant interferometer we have the following equation: $V_{i j}^{\text {obs }}=V_{k(i, j)}^{\text {true }} \cdot G_{i} \cdot G_{j}^{*}+n_{i j}$, with $k=1, \ldots, N_{\text {red }}$ indicating the redundant baselines. There are $N_{\text {tel }}$ unknown complex telescope gains $\left(G_{i}\right)$ plus $N_{\text {red }}$ unknown true visibilities $\left(V_{k}^{\text {true }}\right) ; V_{i j}^{\text {obs }}$ is the observed visibility and $n_{i j}$ is a noise term. The absolute flux scale and position are lost because the product of $G_{i}$ 's and $V_{k}$ 's does not constrain these. Therefore a model of the skybrightness is needed to align all solutions for different hour angles to the same flux scale and position frame.

Redundancy has been used for the Westerbork Synthesis Radio Telescope (WSRT) since about 1980 (Noordam and de Bruyn 1982). The WSRT is a linear East-West array consisting of 10 fixed, equally spaced telescopes and 4 movable telescopes. In the optimal configuration of the WSRT there are 91 interferometers of which 66 have redundancy, there are 14 telescopes gains and 13 unknown true visibilities; the corresponding system of equations is overdetermined and can be solved using e.g., a least squares method.

\section{Advantages of redundancy}

The main advantage of the redundancy solution is that it is model-independent, therefore it has no difficulty with very complex or high dynamic range fields. It also is a one-iteration solution, which means it is relatively fast. In the alignment step only two parameters (gain and phase slope) have to be solved using a skybrightness model, whereas standard selfcal solves $N_{\text {tel }}$ complex parameters. 
Drawbacks of redundancy

In principle redundancy is the perfect method for maximally redundant arrays. But such arrays make rather inefficient use of the available telescopes in terms of U-V-coverage. Most arrays (like the WSRT) are only partially redundant. This means there often are telescopes which occur in only a few redundant interferometers. For these telescopes the gain solutions become relatively noisy and sensitive to interference. The noisy telescope gains result in a noise increase in the corrected visibilities. For the WSRT the movable telescopes can have problematic solutions, the noise increase in the corrected visibilities can reach 20-40\%. Figure 1a shows the rms gains of the 14 telescopes for the optimal configuration $(9 \mathrm{~A}=72 \mathrm{~m})$, a common near-optimal one $(9 \mathrm{~A} \neq 72 \mathrm{~m})$ and the worst case with only 28 redundant baselines (interferometers are traded for extra bandwidth).

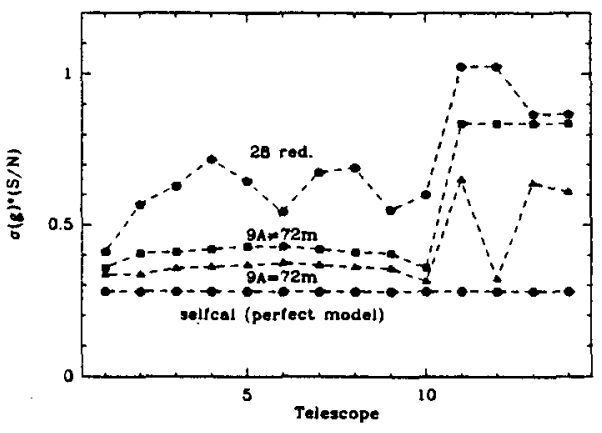

Figure 1. a) The $\mathrm{S} / \mathrm{N}$-scaled $\mathrm{rms}$ of the redundancy gain solutions for 3 WSRT configurations (see text) and for the selfcal solution

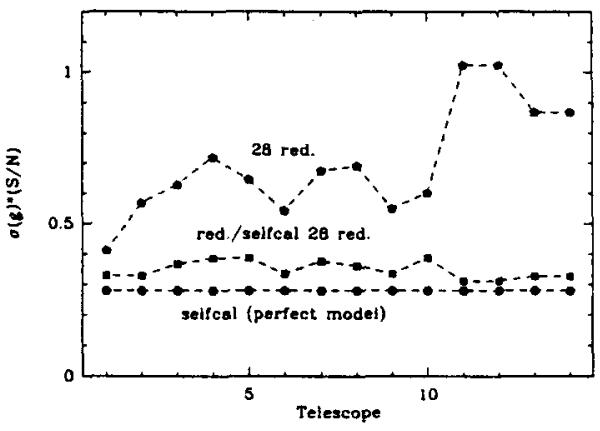

b) The 28 red. solution using the combined redundancy/selfcal approach compared with each separately.

REDUN 9A $\neq 72 \mathrm{~m}$

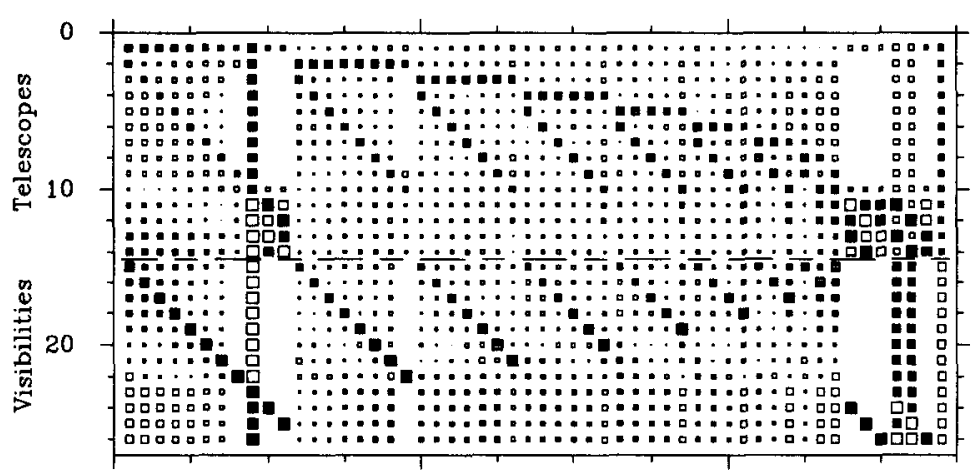

0

20

40

Interferometers

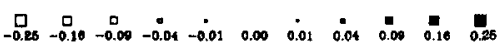

Figure 2. A redundancy gain solution matrix for the WSRT with values represented by symbol type and size. Telescope gains and redundant visibilities are separated by a broken line. 


\section{REDUNDANCY SOLUTION METHOD}

As an example of the solution method we take the case of a 5 telescope linear array of equally spaced telescopes. We will restrict ourselves to the gain solution (taking the logarithm of the equations, with $g=\log (|G|)$ and $v=\log (|V|)$ ) and will use equal weights for all equations (in reality the weights will vary with signal to noise ratio). In this case we have the following equations for the 9 redundant interferometers, the last equation constrains the overall gain. We can write these equations in matrix form as shown on the right hand side.

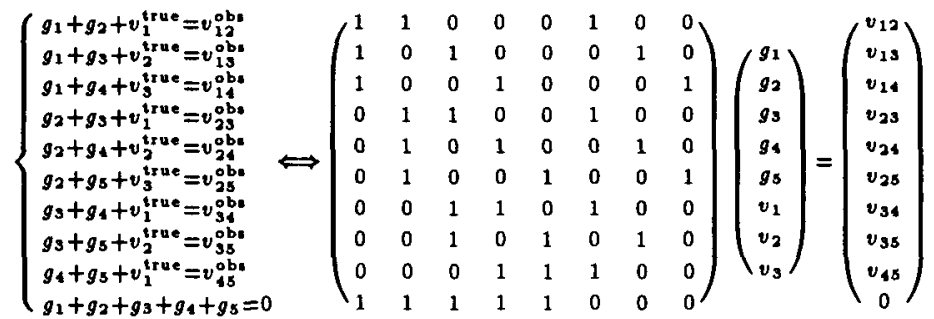

The least squares solution to this matrix equation is given by

$$
\left(\begin{array}{l}
g_{1} \\
g_{2} \\
g_{3} \\
g_{4} \\
g_{3} \\
v_{1} \\
v_{2} \\
v_{3}
\end{array}\right)=\left(\begin{array}{rrrrrrrrrr}
0.37 & 0.27 & 0.17 & -0.20 & -0.20 & -0.17 & -0.20 & -0.07 & 0.03 & 0.20 \\
0.12 & -0.15 & -0.17 & 0.22 & 0.30 & 0.17 & -0.12 & -0.15 & -0.22 & 0.20 \\
-0.30 & 0.10 & 0.00 & 0.30 & -0.20 & 0.00 & 0.30 & 0.10 & -0.30 & 0.20 \\
-0.22 & -0.15 & 0.17 & -0.12 & 0.30 & -0.17 & 0.22 & -0.15 & 0.12 & 0.20 \\
0.03 & -0.07 & -0.17 & -0.20 & -0.20 & 0.17 & -0.20 & 0.27 & 0.37 & 0.20 \\
0.35 & 0.05 & 0.00 & 0.15 & -0.10 & 0.00 & 0.15 & 0.05 & 0.35 & -0.40 \\
0.10 & 0.30 & 0.00 & -0.10 & 0.40 & 0.00 & -0.10 & 0.30 & 0.10 & -0.40 \\
-0.15 & 0.05 & 0.50 & 0.15 & -0.10 & 0.50 & 0.15 & 0.05 & -0.15 & -0.40
\end{array}\right)\left(\begin{array}{l}
v_{12} \\
v_{13} \\
v_{14} \\
v_{23} \\
v_{24} \\
v_{25} \\
v_{34} \\
v_{33} \\
v_{45} \\
0
\end{array}\right)
$$

For the WSRT, in a non-optimal configuration, the corresponding matrix is displayed graphically in figure 2 . For this configuration $(9 A \neq 72 \mathrm{~m})$ there are 12 unknown visibilities and 53 redundant interferometers yielding a $26 \times 54$ solution matrix. The biggest symbols on a given row indicate the interferometers dominating the solution for the corresponding gain or visibility. The more regular the symbol sizes on a given row, the more accurate the corresponding parameter will be estimated. Note the very regular structure for the fixed telescopes (rows 110). In contrast the movable telescopes on rows 11-14 show a few very large values indicating a solution which hinges on only a few interferometers. These solutions will therefore be much more susceptible to noise and interference.

\section{WHY NOT USE PLAIN SELFCAL?}

If redundancy can cause problems for partially redundant arrays, why not use selfcal? Selfcal has the advantages that all data can be used in the solution, that the solutions have good $\mathrm{S} / \mathrm{N}$ and that they are relatively insensitive to interference.

However, selfcal has its drawbacks: it is model dependent and therefore convergence is slow for complex fields and it can be dangerous, especially for small 
arrays. For standard selfcal with one solution per integration time a fraction $\sim 2 / N_{\text {tel }}$ of the data is replaced by model visibilities, due to covariance between data and corrections. This results in artificial noise decrease, appearance of model errors in the map and partial removal of structures not in the model. Most important however, is the fact that not all available information is used if we use standard selfcal for partially redundant arrays.

\section{COMBINED REDUNDANCY/SELFCAL}

For partially redundant arrays we wish to use the redundant information without the drawbacks of possibly increased noise and 'wild' solutions due to interference. The currently available method makes use of the DWARF reduction package for WSRT data. It allows one to improve the 'noisy' telescope gain solutions (those for the 4 movables) using a final, less constrained selfcal which solves for these telescope gains once a good model of the field has been obtained. This technique is currently used as the standard method, it reduces the noise increase by $\sim 50 \%$.

A second method combines the redundancy equations with selfcal equations for the non-redundant baselines. Simulations indicate that the drawbacks of redundancy disappear: the noise propagation and the sensitivity to interference become comparable to selfcal; on the other hand the solutions are less model dependent than with selfcal. The relative weights of the selfcal and redundancy equations can be adjusted according to the model completeness, giving the selfcal equations lower weight while the model is still incomplete. The effect of this method on the rms of the gain solutions is shown in figure $1 \mathrm{~b}$, where the worst case of figure 1a has now been solved using the combined method, using equal weights for both sets of equations.

\section{CONCLUSIONS}

We conclude that redundancy should be used when available but one should be aware of possible problems with interference and low $\mathrm{S} / \mathrm{N}$ data. The use of selfcal for small arrays can be dangerous, partially transforming the data into the model. The combined redundancy/selfcal method looks promising for partially redundant arrays: it combines the best of both methods with the possibility to optimize the balance between the redundancy and selfcal components according to model completeness.

\section{ACKNOWLEDGEMENTS}

The author thanks Wim Brouw, Ger de Bruyn, Peter Katgert and Rudolf Le Poole for useful comments and suggestions. Shell Nederland provided a travel grant and the Leids Kerkhoven-Bosscha fonds provided financial support.

\section{REFERENCE}

Noordam, J.E., de Bruyn, A.G., 1982, Nature, 5884, 597. 
Tim Cornwell: The real test of your conclusions is whether you would recommend redundancy for a new array. If so, how much redundancy is necessary? A comment: I would rephrase your statement 'selfcal is dangerous for small $N$ ' to 'imaging is dangerous for small $N$ '. We are interested in the properties of the final image not the intermediate gain solutions.

Mark Wieringa: Yes, but there always is a tradeoff between $u-v$ coverage and the amount of redundancy. I have not looked into this question in any detail, but I think the amount of redundancy needed depends on the expected errors that have to be corrected.

Peter Dewdney: In the situation in which you are using a combined redundancy/self-cal solution, is the role of the 'redundancy part' to improve the final solution or to find the solution faster? In other words, is the final image the same using the method of self-cal and redundancy as using the method of self-cal alone?

Mark Wieringa: My initial response is yes. They will be the same and using redundancy just speeds up the calibration considerably. But this is very much dependent on the complexity of the field, the $u-v$ coverage and the magnitude of the errors: pure selfcal may fail to converge to an accurate solution when the errors are too large and the coverage too sparse.

John Baldwin: To emphasize Tim Cornwell's point, the important question is: given a number of telescopes how should one site them to use the maximum astronomical information. Do you agree that we still have no answer? Mark Wieringa: Yes, I agree, the answer will depend on the complexity of the field and the magnitude of the telescope errors. When large they may prevent an accurate selfcal solution. Making use of redundancy will often solve this.

Ron Ekers: There are two separate issues being confused: i) the advantages of having redundant spacings and ii) the best algorithm to use if you have redundant spacings.

Ron Ekers: Does a fully redundant one-dimensional array used for earth rotation synthesis still have a unique $2 \mathrm{D}$ solution? Note that there is no redundancy in the "time direction".

Mark Wieringa: (No written response was provided) 